Mots. Les langages du politique

$116 \mid 2018$

Dire ou ne pas dire la « race » en France aujourd'hui

\title{
Jean-Marc Leblanc, Analyses lexicométriques des vœux présidentiels
}

Londres, ISTE éditions, 2016

Michèle Monte

\section{OpenEdition}

\section{Journals}

Édition électronique

URL : https://journals.openedition.org/mots/23110

DOI : $10.4000 /$ mots. 23110

ISSN : 1960-6001

Éditeur

ENS Éditions

Édition imprimée

Date de publication : 20 mars 2018

Pagination : 148-152

ISSN : 0243-6450

Référence électronique

Michèle Monte, "Jean-Marc Leblanc, Analyses lexicométriques des vœux présidentiels », Mots. Les langages du politique [En ligne], 116 | 2018, mis en ligne le 23 février 2018, consulté le 23 avril 2022.

URL : http://journals.openedition.org/mots/23110; DOI : https://doi.org/10.4000/mots.23110

Ce document a été généré automatiquement le 23 avril 2022.

(c) ENS Éditions 


\section{Jean-Marc Leblanc, Analyses lexicométriques des vœux présidentiels}

Londres, ISTE éditions, 2016

Michèle Monte

\section{RÉFÉRENCE}

Jean-Marc Leblanc, Analyses lexicométriques des vœeux présidentiels, Londres, ISTE éditions, $2016,386 \mathrm{p}$.

1 Ce livre de près de 400 pages est disponible en deux versions, papier et électronique, et il est augmenté de 45 annexes consultables sur le site http://textopol.u-pec.fr/ouvrage. Il analyse les messages de vœux aux Français des présidents de la $\mathrm{V}^{\mathrm{e}}$ République, diffusés le 31 décembre chaque année. Ce corpus présente ainsi une belle ampleur chronologique et une homogénéité générique qui permettent des comparaisons fructueuses. En revanche, comme l'indique le premier chapitre méthodologique, on ne peut extrapoler de ces courts textes rituels des analyses qui concerneraient l'ensemble du discours présidentiel. Le lecteur aura ainsi intérêt à comparer les résultats présentés dans ce livre aux analyses plus globales proposées par Dominique Labbé (1990), Dominique Labbé et Denis Monière (2003), Damon Mayaffre (2004 et 2012), d'ailleurs convoquées en plusieurs endroits du livre à titre de contrepoint.

2 Le chapitre introductif brosse un historique des analyses des discours présidentiels assistées par ordinateur et définit le discours de vœux comme un « discours épidictique au style élevé » (p. 27), puis il précise les objectifs du livre en insistant sur les principes de l'analyse lexicométrique : la norme par rapport à laquelle les textes sont comparés est endogène, constituée par le corpus dans sa globalité, le travail effectue des allersretours constants entre les sorties machine et le texte pour contextualiser les formes qui appellent l'analyse. Enfin, le premier chapitre présente à grands traits les principaux logiciels qui seront mobilisés au fil du livre: Lexico 3, Hyperbase, TXM/ Weblex, Tropes, Alceste, et les types de mesures qui seront effectuées au moyen de ces 
outils. Il se termine par la présentation de l'outil de visualisation TextObserver qui permet de faire varier sous nos yeux les résultats des analyses factorielles de correspondances (AFC) en modifiant les données du calcul.

Le chapitre II intitulé « Éthos et diachronie » est orienté par une question extrêmement pertinente: les contrastes observés relèvent-ils plutôt de l'éthos de chacun des présidents concernés ou de l'évolution chronologique et des évènements qui ont marqué la période ? Il analyse d'abord les septennats (1959-2001). Les résultats des AFC par années ainsi que les mesures des distances entre textes et entre vocabulaires, audelà de différences dues à la méthode de calcul, montrent la singularité du lexique de De Gaulle, Chirac et Mitterrand, la plus grande hétérogénéité du lexique de Giscard et Pompidou. L'étude des spécificités met en évidence le poids de l'évènementiel, mais aussi de thématiques et d'éthos personnels : de Gaulle utilise des formes qui articulent les énoncés entre eux ou qui argumentent (du point de vue de, notamment, sans doute). Le vocabulaire des vœux est très abondant chez Giscard. Chirac recourt à un vocabulaire mobilisateur et volontaire. Mitterrand évoque souvent l'Europe. Cependant l'analyse arborée de la distance lexicale sur un corpus étendu aux quinquennats confirme le poids de l'évolution chronologique, que l'on peut sans doute corréler à l'évolution des mœurs politiques.

4 Après cette vue d'ensemble, le chapitre III propose une analyse fine des données morphosyntaxiques et énonciatives sur la période 1959-2001. La centralité de Chirac observée par D. Mayaffre sur un corpus multigénérique est visible sur le corpus vœux étiqueté, ce qui n'était pas le cas sur le corpus non catégorisé. Le corpus vœux confirme également la tendance à l'accroissement des verbes et du présent de l'indicatif au fil du temps par rapport aux autres catégories grammaticales. Les partitions par années permettent souvent de nuancer ce qu'une partition par locuteurs aurait de trop global et d'observer que la première année du septennat et celles qui sont marquées par des échéances électorales ont tendance à se singulariser. De ce fourmillement de résultats accompagnés de précieux retours au texte, je ne retiendrai que quelques aspects parmi les plus saillants: le goût de Chirac pour les déontiques et l'infinitif, le rôle de l'imparfait pour évoquer des souvenirs personnels, la variété des valeurs du futur, tour à tour promissif, directif, ou métadiscursif, ce qui invite à ne pas s'en tenir aux purs résultats quantitatifs. Les contrastes dans l'emploi des marques personnelles sont, quant à eux, spectaculaires : de Gaulle sous-emploie le je et le vous, que Giscard et, dans une moindre mesure, Pompidou suremploient. Mitterrand se caractérise par un suremploi du je et du on, alors que Chirac use légèrement plus du nous que la moyenne et emploie de moins en moins le je au fil du temps, se rapprochant ainsi de De Gaulle. Enfin, l'étude de l'accroissement spécifique du vocabulaire montre l'essor du lexique économique lié à la croissance, au chômage et à l'emploi, le déclin de celui qui est lié au progrès et au développement, la progression du lexique des valeurs républicaines. L'étude du lexème sécurité (p. 154-155) montre l'importance capitale du contexte pour une interprétation correcte de l'usage d'un mot dans un discours. Le renouvellement lexical est plus le fait d'années spécifiques que d'un mouvement continu, même si on constate une forte stabilité du vocabulaire avec Chirac.

5 Le chapitre IV affine la description de l'éthos présidentiel en étudiant les cooccurrents $\mathrm{du}$ je, du vous et du nous. Parmi les verbes qui accompagnent le je, Jean-Marc Leblanc analyse très finement l'emploi de penser qui est souvent un marqueur affectif, de savoir qui est le support de stratégies concessives ou de manifestations d'empathie. 
Différentes méthodes (lexicogrammes dans TXM, tri croisé d'Alceste) montrent qu'une polarité se dessine entre les passages en je marqués par le rituel des vœux et l'expression de l'empathie et les passages sans je, où domine le lexique politique, économique et social. L'étude du nous mobilise la méthode des poly-cooccurrences: autour des verbes modaux et d'action s'agrègent des noms tels que société, marché, paix, Europe, des participes tels qu'engagés ou respectés. Dans l'interaction entre je et vous, ce qui transparait, c'est l'éthos du guide qui «explique, clarifie son action et son discours» (p. 217) et la construction d'une relation de proximité marquée par des expressions telles que " je sais que vous », « je pense comme vous que », « il y a un an, je vous disais ", relation qui se développe à partir de Pompidou.

6 Le chapitre $v$, après un rapide parcours des résultats obtenus avec Tropes, effectue une analyse thématique basée sur la classification hiérarchique descendante d'Alceste (implémentée également dans Iramuteq). Cette classification qui retient $61 \%$ des énoncés du corpus dégage cinq classes thématiques :

- la classe du rituel des vœux, à laquelle Giscard contribue fortement et qui contient les termes d'adresse, les verbes de souhait et beaucoup de marques personnelles ;

- la classe relative à la politique internationale, à laquelle de Gaulle et Mitterrand contribuent beaucoup, et qui regroupe les noms de pays et des mots tels qu'Union européenne, détente, indépendance, défense, tiers-monde;

- la classe des valeurs et de la mobilisation, surreprésentée chez Chirac;

- la classe de la politique intérieure, économique et sociale, surreprésentée chez de Gaulle ;

- une classe plus hétérogène regroupant des mots désignant la situation de la France face au monde et à l'avenir.

7 L'analyse par locuteurs permet ensuite d'affiner les résultats : en effet, si on retrouve à peu près les mêmes classes thématiques chez les cinq présidents, leur importance relative et leur composition interne varient. Alors que les thématiques de De Gaulle sont très variées, avec un accent particulier sur le progrès technico-scientifique et le développement d'une part, sur les relations Est-Ouest et Europe-Amérique d'autre part, Pompidou se distingue par une classe où il évalue son action et une autre où il associe grandeur, prospérité et référence à de Gaulle; on observe chez Giscard une classe où il analyse les difficultés et atouts de la France face à un monde en mutation, chez Mitterrand un développement important de la politique internationale. Quant à Chirac, ses énoncés comportent une classe où le devoir de l'État est affirmé avec insistance c'est à lui qu'il incombe de faire respecter la loi, l'autorité, la cohésion nationale - et une autre où le lexique économique et social s'associe à un lexique de lutte et d'action.

Le dernier chapitre s'intéresse aux nouveautés observables dans les discours de Hollande et Sarkozy. Du point de vue méthodologique, il nous alerte sur le fait que l'accroissement du corpus modifie les équilibres internes : ainsi la classe du rituel se scinde-t-elle en deux, l'une autour des termes d'adresse, l'autre autour de l'empathie avec ceux qui souffrent, la classe du vocabulaire économique et social prend de l'ampleur, abondée par Hollande, alors que la classe de la politique internationale régresse en poids. Sarkozy vient abonder la classe des valeurs jusqu'alors surtout incarnée par Chirac. L'étude année après année fait ressortir, quant à elle, la variabilité des vœux de Sarkozy, d'abord à l'exact opposé de ceux de De Gaulle, puis se rapprochant peu à peu d'une position moyenne, et la proximité entre le lexique de Sarkozy et celui de Hollande. On peut regretter cependant le manque de clarté de ce 
chapitre qui piétine en revenant sur des éléments déjà bien établis dans les chapitres antérieurs et qui ne se centre pas assez sur les spécificités des derniers présidents.

Ce livre n'offre pas de révélations foudroyantes sur le langage des présidents, il vient plutôt confirmer, sur un genre particulier qui est le discours de vœux, des observations déjà faites dans d'autres travaux, tout en montrant comment les règles du genre influencent la construction du discours et impactent les sorties machine. Mais il se révèle très précieux sur le plan méthodologique: les biais de chaque méthode sont expliqués très clairement, les interprétations des graphiques et tableaux sont menées avec beaucoup de prudence, confrontées entre elles et relativisées, parfois à l'excès. L'ouvrage souffre en effet des défauts de ses qualités : très minutieux et pédagogique, il sacrifie un peu trop la synthèse à l'analyse, surtout, me semble-t-il, dans les deux derniers chapitres qui n'offrent pas toujours la montée en puissance interprétative attendue. Mais le livre de J.-M. Leblanc vient, sur un exemple concret, combler un vide en montrant comment mener des analyses outillées, avec quelles méthodes. La part à accorder aux différentes variables dans l'interprétation - poids des évènements, évolution des mœurs politiques, éthos des locuteurs - est présentée avec beaucoup de rigueur. L'ouvrage fait comprendre aux novices comment on construit pas à pas une interprétation en faisant parler les chiffres et les graphiques, mais aussi en s'entourant des précautions nécessaires. Il abonde en exemples que pourront utiliser tous ceux et celles qui forment leurs étudiants à de tels outils, et il offre aux doctorants qui se lancent dans de telles analyses un questionnement, un recul critique, une clarté et une finesse d'analyse dont ils pourront s'inspirer pour leurs propres recherches.

\section{BIBLIOGRAPHIE}

LABBÉ Dominique, 1990, Le vocabulaire de François Mitterrand, Paris, Presses de la Fondation nationale des sciences politiques.

LABBÉ Dominique, MONIÈRE Denis, 2003, Le discours gouvernemental. Canada, Québec, France (1945-2000), Paris, Honoré Champion.

MAYAFFRE Damon, 2012, Nicolas Sarkozy. Mesure et démesure du discours (2007-2012), Paris, Presses de Sciences Po.

MAYAFFRE Damon, 2004, Paroles de président. Jacques Chirac (1995-2003) et le discours présidentiel sous la $V^{e}$ République, Paris, Honoré Champion.

\section{AUTEURS}

\section{MICHĖLE MONTE}

Université de Toulon 